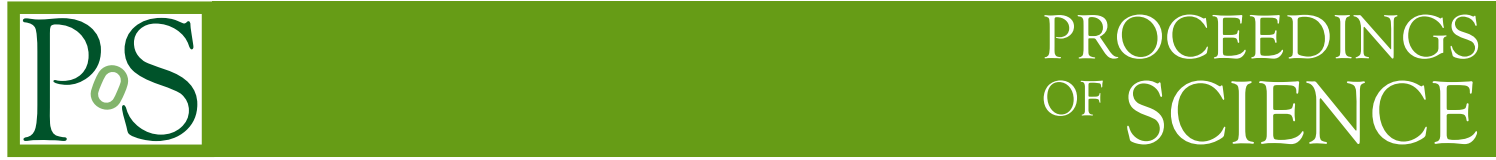

\title{
Recent Bottomonium Results from BaBar and Belle
}

\author{
Veronique Ziegler*十 \\ SLAC National Accelerator Laboratory \\ E-mail: vziegler@slac.stanford.edu
}

Selected studies in bottomonium physics carried out by the BaBar and Belle experiments are presented. They include a study of radiative bottomonium transitions using $\gamma \rightarrow e^{+} e^{-}$conversions done by BaBar, the search for the $h_{b}(1 P)$ and $h_{b}(2 P)$ states leading to an evidence for the $h_{b}(1 P)$ seen by BaBar in $\Upsilon(3 S) \rightarrow \pi^{0} h_{b}$ decay and to the Belle observation of both the $h_{b}(1 P)$ and the $h_{b}(2 P)$ in the reaction $e^{+} e^{-} \rightarrow \pi^{+} \pi^{-} h_{b}(1 P, 2 P)$ from $\Upsilon(5 S)$ data.

The 13th International Conference on B-Physics at Hadron Machines, BEAUTY 2011, April 4-8 2011

Amsterdam, The Netherlands

\footnotetext{
* Speaker.

${ }^{\dagger}$ On behalf of the BaBar and Belle Collaborations.
} 


\section{Introduction}

Bottomonia are the heaviest of the $q \bar{q}$ bound states. Decays within the bottomonium family of states occur via $\pi^{0}, \eta, \omega$ or di-pion emission, or by electric dipole transitions or magnetic dipole transitions. Electromagnetic transitions between the energy levels of the bottomonium spectrum can be calculated in the quark model and are an important tool in understanding the internal structure of $b \bar{b}$ bound states. In particular, the measurement of the hyperfine mass splittings between triplet and singlet $P$-wave states is of key importance in understanding the role of spin-spin interactions in quarkonium models, and in testing QCD calculations. In the non-relativistic approximation, the hyperfine splitting is proportional to the square of the wave function at the origin, which is expected to be non-zero only for $S$-wave states (i.e. for $L=0$, where $L$ is the orbital angular momentum quantum number of the $q \bar{q}$ system).

In particular, the mass splitting between the $\Upsilon(1 S)$ and the $\eta_{b}(1 S)$ is a key ingredient in many theoretical calculations. The value measured by BaBar, $m(\Upsilon(1 S))-m\left(\eta_{b}(1 S)\right)=69.3 \pm 2.8$ $\mathrm{MeV} / \mathrm{c}^{2}[1,2]$, and subsequently by CLEO [3] is larger than most predictions based on potential models [4], but is in reasonable agreement with predictions from lattice calculations [5]. According to ref. [6], the shift in measured $\eta_{b}(1 S)$ compared to QCD predictions could be explained by the mixing of the $\eta_{b}$ with a CP-odd Higgs scalar. To test this model, a measurement of the $\eta_{b}$ width is essential, and was one motivation for the BaBar search for the $\eta_{b}$ using photon conversions, as described in Sec. 2.

For $L=1$, the mass splitting between the spin-singlet $\left({ }^{1} P_{1}\right)$ and the spin-averaged triplet state $\left(\left\langle{ }^{3} P_{J}\right\rangle\right)$ is expected to be $\Delta M_{\mathrm{HF}}=M\left({ }^{3} P_{J}\right)-M\left({ }^{1} P_{1}\right) \sim 0$. The ${ }^{1} P_{1}$ state of bottomonium, the $h_{b}(1 P)$, is the axial vector partner of the $P$-wave $\chi_{b J}(1 P)$ states. Its expected mass, computed as the spinweighted center of gravity of the $\chi_{b J}(1 P)$ states [7], is $9899.87 \pm 0.27 \mathrm{MeV} / c^{2}$. Higher-order corrections might cause a small deviation from this value, but a hyperfine splitting larger than 1 $\mathrm{MeV} / \mathrm{c}^{2}$ might be indicative of a vector component in the confinement potential [8]. The hyperfine splitting for the charmonium ${ }^{1} P_{1}$ state $h_{c}$ is measured by the BES and CLEO experiments $[9,10,11]$ to be $\sim 0.1 \mathrm{MeV} / \mathrm{c}^{2}$. An even smaller splitting is expected for the much heavier bottomonium system [8].

Both the BaBar [12] and Belle [13] experiments collected data at the narrow $\Upsilon(n S)$ resonances and above the $\Upsilon(4 S)$ in order to carry out detailed studies in bottomonium spectroscopy ${ }^{1}$.

In this paper, we summarize the results of a study of radiative bottomonium transitions using $\gamma \rightarrow e^{+} e^{-}$conversions done by BaBar, the search for the $h_{b}(1 P)$ and $h_{b}(2 P)$ states leading to an evidence for the $h_{b}(1 P)$ seen by BaBar in $\Upsilon(3 S) \rightarrow \pi^{0} h_{b}(1 P)$ decay [14] and the Belle observation of both the $h_{b}(1 P)$ and the $h_{b}(2 P)$ in the reaction $e^{+} e^{-} \rightarrow \pi^{+} \pi^{-} h_{b}(1 P, 2 P)$ from $\Upsilon(5 S)$ data [15].

\section{Study of radiative bottomonium transitions using $\gamma \rightarrow e^{+} e^{-}$conversions}

We summarize the results of a study of radiative transitions in the bottomonium system using the inclusive $\gamma \rightarrow e^{+} e^{-}$energy spectrum from $\Upsilon(3 S)$ and $\Upsilon(2 S)$ decays. The rate of $\gamma \rightarrow e^{+} e^{-}$

\footnotetext{
${ }^{1}$ The BaBar samples correspond to $14 \mathrm{fb}^{-1}$ of $\Upsilon(2 S)$ data and $30 \mathrm{fb}^{-1}$ of $\Upsilon(3 S)$ data, and to $3.2 \mathrm{fb}^{-1}$ of scanned data taken near the $\Upsilon(5 S)$ resonance. The Belle samples consist of $6,24,3$, and $121 \mathrm{fb}^{-1}$ of $\Upsilon(1 S), \Upsilon(2 S), \Upsilon(3 S)$, and $\Upsilon(5 S)$ data, respectively.
} 

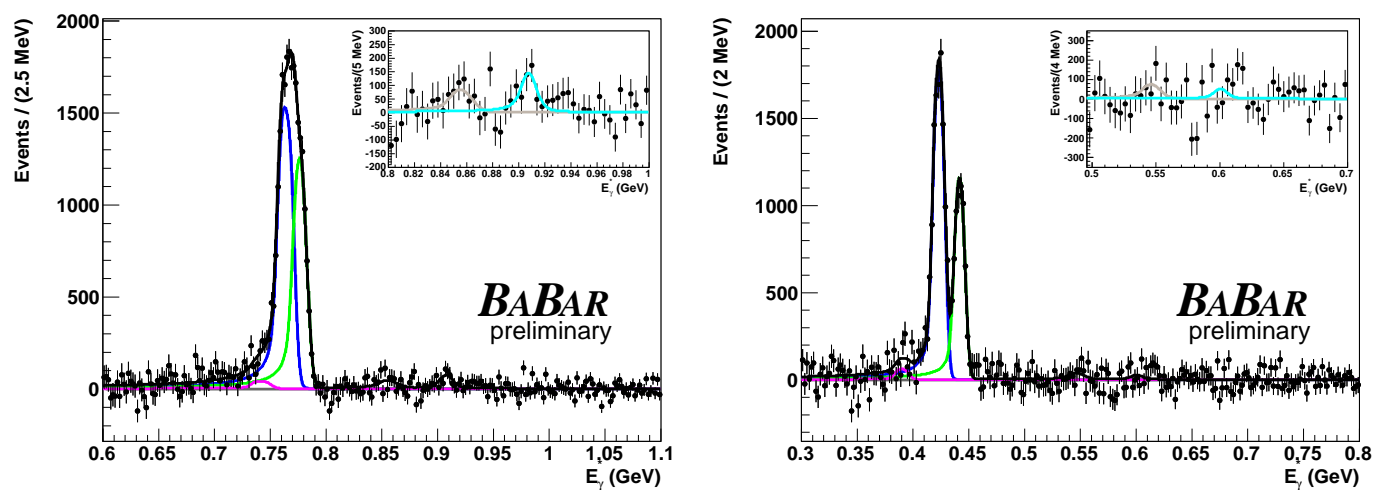

Figure 1: Fit results for the inclusive converted photon spectrum after background-subtraction for (left) $\Upsilon(3 S)$, and (right) $\Upsilon(2 S)$ data. The purple, blue, green, gray, and cyan curves represent (left) $\chi_{b 0,1,2}(2 P) \rightarrow$ $\gamma \Upsilon(1 S)$ and (right) $\chi_{b 0,1,2}(1 P) \rightarrow \gamma \Upsilon(1 S)$, initial state radiation (ISR), and $\Upsilon(n S) \rightarrow \gamma \eta_{b}(1 S)$ events, respectively.

conversion in the detector material, and of the reconstruction of the $e^{+} e^{-}$pairs is much lower than that for photons reconstructed using the BaBar electromagnetic calorimeter. However, the substantial improvement ${ }^{2}$ in energy resolution for photon conversions results in better separation of photon energy lines.

The decay $\Upsilon(3 S) \rightarrow \gamma \chi_{b 0,2}(1 P)$ is observed, and precise measurements of the branching fractions for $\chi_{b 1,2}(2 P) \rightarrow \gamma \Upsilon(2 S)$ and $\chi_{b 1,2}(1 P, 2 P) \rightarrow \gamma \Upsilon(1 S)$ decays obtained. The backgroundsubtracted fit results for the latter are shown in Fig. 1. The product of branching fractions $\mathscr{B}(\Upsilon(n S) \rightarrow$ $\left.\gamma \chi_{b J}\right) \times \mathscr{B}\left(\chi_{b J} \rightarrow \Upsilon(1 S)\right)$ obtained from the fit of Fig. 1 are consistent with and improve upon the current values [7].

The searches for $\eta_{b}(1 S)$ and $\eta_{b}(2 S)$ states using the converted photon energy spectrum are inconclusive. Over a range of approximately $9974<m_{\eta_{b}(2 S)}<10015 \mathrm{MeV} / c^{2}$, we find $\mathscr{B}(\Upsilon(3 S) \rightarrow$ $\left.\gamma \eta_{b}(2 S)\right)<1.9 \times 10^{-3}$ (at $90 \%$ C.L.). This value is consistent with, but does not improve upon, the upper limit obtained by CLEO [16]. Due to low efficiency and high background levels, no evidence for $\Upsilon(2 S) \rightarrow \gamma \eta_{b}(1 S)$ is found. The most significant peaking structure seen in the $e^{+} e^{-}$ center-of-mass energy $\left(E^{*}(\gamma)\right)$ region expected for the $\Upsilon(3 S) \rightarrow \gamma \eta_{b}(1 S)$ transition, if interpreted as an $\eta_{b}(1 S)$ signal, trends toward recent potential model [17] and lattice [18] predictions. However, the small $(<3 \sigma)$ significance of the result is insufficient to measure the $\eta_{b}(1 S)$ mass in the present analysis. Taking advantage of the improved resolution from a converted photon technique to make a definitive measurement of the $\eta_{b}(1 S)$ mass and width will require much more data from future experiments.

\section{The BaBar search for $h_{b}(1 P)$ in $\Upsilon(3 S)$ data}

The $h_{b}(1 P)$ state is expected to be produced in $\Upsilon(3 S)$ decay via $\pi^{0}$ or di-pion emission, and to undergo a subsequent $E 1$ transition to the $\eta_{b}(1 S)$, with branching fraction $(\mathrm{BF}) \mathscr{B}\left(h_{b}(1 P) \rightarrow\right.$

\footnotetext{
${ }^{2}$ e.g. from $\sim 25 \mathrm{MeV}$ in the calorimeter to $4 \mathrm{MeV}$ or better with converted photons
} 

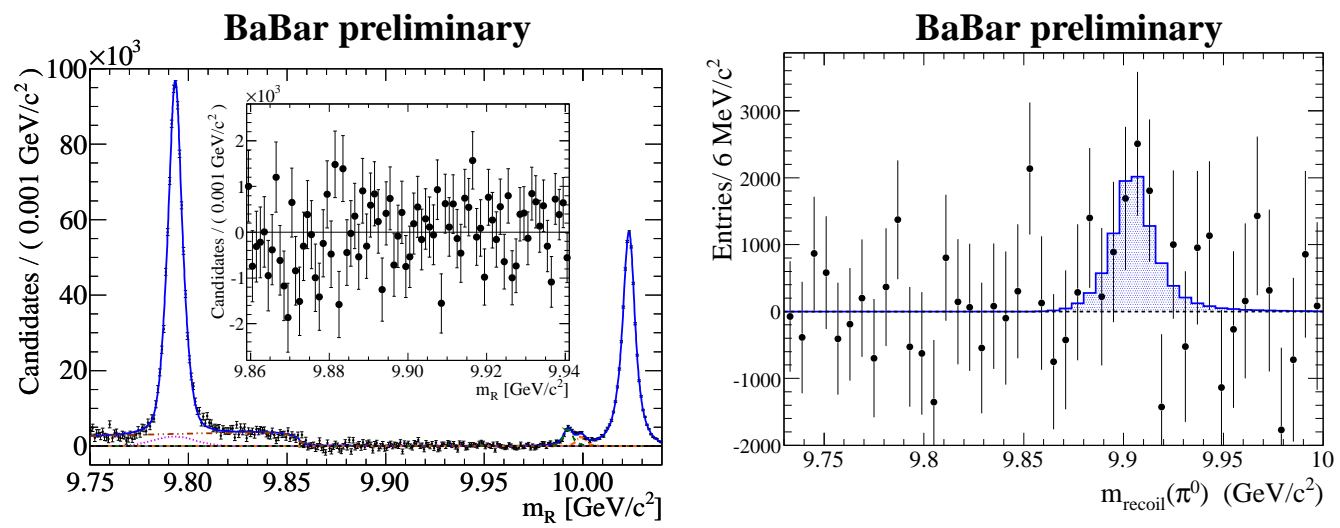

Figure 2: Results of the search for $\Upsilon(3 S) \rightarrow \pi^{+} \pi^{-} h_{b}(1 P)$ (left) and $\Upsilon(3 S) \rightarrow \pi^{0} h_{b}(1 P)$ (right). (left) The $m_{R}$ spectrum after subtraction of the continuum background component. The curves represent the $\Upsilon_{I S R}^{2 \rightarrow 1}$ (dotted), $K_{S}^{0}$ (double-dot-dashed), $\chi_{b}^{1,1}$ (dashed), and $\chi_{b}^{2,2}$ (dot-dashed) components. Inset: expanded view in the $h_{b}$ region after subtraction of continuum and peaking backgrounds. (right) The $m_{\text {recoil }}\left(\pi^{0}\right)$ spectrum after subtracting background; the shaded histogram represents the signal function resulting from the fit to the data.

$\left.\gamma \eta_{b}(1 S)\right) \sim(40-50) \%$ [8]. The isospin-violating decay $\Upsilon(3 S) \rightarrow \pi^{0} h_{b}(1 P)$ is expected to have a BF of about $0.1 \%[19,20]$, while theoretical predictions for the transition $\Upsilon(3 S) \rightarrow \pi^{+} \pi^{-} h_{b}(1 P)$ range from $\sim 10^{-4}[19]$ up to $\sim 10^{-3}$ [21]. The CLEO experiment reported the $90 \%$ C.L. limit $\mathscr{B}\left(\Upsilon(3 S) \rightarrow \pi^{0} h_{b}(1 P)\right)<0.27 \%$ [22] based on fewer than 0.5 million $\Upsilon(3 S)$ events.

We search for a signal in the inclusive recoil mass distribution against di-pion $\left(m_{R}\right)$ or $\pi^{0}$ $\left(m_{\text {recoil }}\left(\pi^{0}\right)\right)$ candidates.

No $h_{b}(1 P)$ signal is seen in the $\Upsilon(3 S) \rightarrow \pi^{+} \pi^{-} h_{b}$ production channel. Assuming the $h_{b}$ mass to be $9.900 \mathrm{GeV} / c^{2}$, we set a $90 \%$ C.L. upper limit $\mathscr{B}\left[\Upsilon(3 S) \rightarrow \pi^{+} \pi^{-} h_{b}\right]<1.2 \times 10^{-4}$. The branching fraction measurement $\mathscr{B}\left[\Upsilon(3 S) \rightarrow \pi^{+} \pi^{-} \Upsilon(2 S)\right]=(3.00 \pm 0.02$ (stat.) \pm 0.14 (syst.) $) \%$ is more precise than the current world average $(2.45 \pm 0.23) \%$ [7]. Branching fractions $\mathscr{B}\left[\chi_{b 1}(2 P) \rightarrow\right.$ $\left.\pi^{+} \pi^{-} \chi_{b 1}(1 P)\right]=(9.2 \pm 0.6 \pm 0.9) \times 10^{-3}, \mathscr{B}\left[\chi_{b 2}(2 P) \rightarrow \pi^{+} \pi^{-} \chi_{b 2}(1 P)\right]=(4.9 \pm 0.4 \pm 0.6) \times$ $10^{-3}$, and $\mathscr{B}[\Upsilon(3 S) \rightarrow X \Upsilon(2 S)] \times \mathscr{B}\left[\Upsilon(2 S) \rightarrow \pi^{+} \pi^{-} \Upsilon(1 S)\right]=(1.78 \pm 0.02 \pm 0.11) \%$ are also obtained from this analysis.

Evidence for the $h_{b}(1 P)$ state in the decay $\Upsilon(3 S) \rightarrow \pi^{0} h_{b}(1 P)$ is found by requiring a photon with an energy consistent with that for the $h_{b}(1 P) \rightarrow \gamma \eta_{b}(1 S)$ transition. The number of $\pi^{0}$ events in $m_{\text {recoil }}\left(\pi^{0}\right)$ is determined from a fit to the $m_{\gamma \gamma}$ distribution in each $m_{\text {recoil }}\left(\pi^{0}\right)$ bin. The resulting distribution contains a broad peaking signal component above a smooth background, as seen in Figure 2. The fit to this distribution shows preliminary evidence (at the $3.0 \sigma$ level) for a $h_{b}(1 P)$ signal, leading to a branching fraction of $\mathscr{B}\left(\Upsilon(3 S) \rightarrow \pi^{0} h_{b}(1 P)\right)=(3.7 \pm 1.1 \pm 0.4) \times 10^{-4}$ corresponding to $90 \%$ C.L. upper limit of $\mathscr{B}\left(\Upsilon(3 S) \rightarrow \pi^{0} h_{b}(1 P)\right)<5.8 \times 10^{-4}$. This measurement would indicate that the $\Upsilon(3 S) \rightarrow \pi^{+} \pi^{-} h_{b}(1 P)$ is suppressed by a factor greater than 3 w.r.t. $\Upsilon(3 S) \rightarrow \pi^{0} h_{b}(1 P)$. The measured mass value, $m\left[h_{b}(1 P)\right]=9902 \pm 4$ (stat.) \pm 2 (syst.) $\mathrm{MeV} / c^{2}$, is consistent with the expectation for the $h_{b}(1 P)$ bottomonium state [8], the axial vector partner of the $\chi_{b J}(1 P)$ triplet of states. 


\section{Belle preliminary}

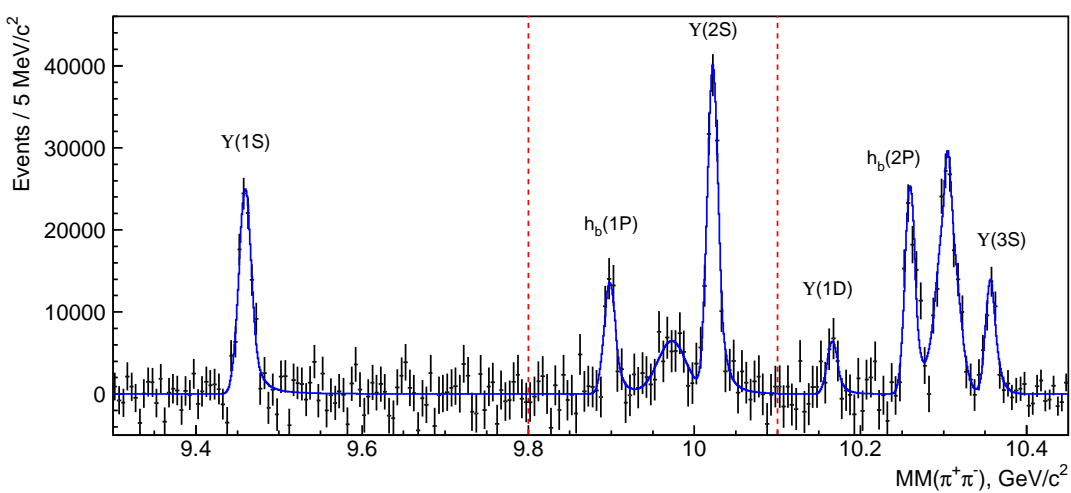

Figure 3: The $M M\left(\pi^{+} \pi^{-}\right)$spectrum with the combinatorial background and $K_{S}^{0}$ contributions subtracted (dots with error bars) and signal components of the fit function (solid histogram). The vertical dashed lines indicate the boundaries of the fit regions. The peaks centered at $9.97 \mathrm{GeV} / c^{2}$ and $10.30 \mathrm{GeV} / c^{2}$ correspond to transitions $\Upsilon(3 S) \rightarrow \Upsilon(1 S) \pi^{+} \pi^{-}$and $\Upsilon(2 S) \rightarrow \Upsilon(1 S) \pi^{+} \pi^{-}$, respectively.

\section{The Belle observation of the $h_{b}(1 P, 2 P)$ states from $\Upsilon(5 S)$ data}

The Belle Collaboration has collected a large sample of $e^{+} e^{-}$events at energy corresponding to the $\Upsilon(5 S)$ resonance, which lies above the threshold for production of $B_{s}$ meson pairs, for the primary purpose of studying $B_{s}$ decays. The observation of anomalously large rates for dipion transitions to lower bottomonium states $\Upsilon(5 S) \rightarrow \Upsilon(n S) \pi^{+} \pi^{-}(n=1,2,3)$ [23] led to the speculation of the existence of an exotic resonance $Y_{b}$ in the vicinity of the $\Upsilon(5 S)$ that might be responsible for the high transition rates [24]. Such a resonance might be an analog, in the bottomonium sector, of the charmonium-like $Y(4260)$. A recent indication by the CLEO Collaboration that the process $e^{+} e^{-} \rightarrow h_{c}(1 P) \pi^{+} \pi^{-}$might have higher transition rate at $Y(4260)$ energy than at $\sqrt{s}=4170 \mathrm{MeV}$ [25] motivated the Belle Collaboration to search for the $h_{b}(n P)$ in $\Upsilon(5 S)$ data, to see if $h_{b}(n P)$ production might be enhanced in the region of the $Y_{b}$.

The Belle Collaboration reports the observation of the $h_{b}(1 P)$ and $h_{b}(2 P)$ bottomonium states produced in the reaction $e^{+} e^{-} \rightarrow h_{b}(n P) \pi^{+} \pi^{-}$with significances of $5.5 \sigma$ and $11.2 \sigma$, respectively, using a sample of $121.4 \mathrm{fb}^{-1}$ of data collected near the peak of the $\Upsilon(5 S)$ resonance $(\sqrt{s} \sim$ $10.865 \mathrm{GeV})$. The measured masses $m\left[h_{b}(1 P)\right]=\left(9898.25 \pm 1.06_{-1.07}^{+1.03}\right) \mathrm{MeV} / c^{2}$ and $m\left[h_{b}(2 P)\right]=$ $\left(10259.76 \pm 0.64_{-1.03}^{+1.43}\right) \mathrm{MeV} / c^{2}$, are consistent with the center-of-gravity of the corresponding $\chi_{b J}$ states.

The measured cross sections relative to the $e^{+} e^{-} \rightarrow \Upsilon(2 S) \pi^{+} \pi^{-}$cross section are large, indicating an anomalous production rate for the $h_{b}(1 P)$ and $h_{b}(2 P)$ and suggesting that these states are produced via an exotic process that violates the suppression of heavy quark spin-flip.

\section{References}

[1] B. Aubert et al. (BaBar Collaboration), Phys. Rev. Lett. 101, 071801 (2008).

[2] B. Aubert et al. (BaBar Collaboration), Phys. Rev. Lett. 103, 161801 (2009). 
[3] G. Bonvicini et al. (CLEO Collaboration), Phys. Rev. D 81, 031104(R) (2010).

[4] For a comprehensive review, see N. Brambilla et al., Eur.Phys.J.C 71, 1534 (2011).

[5] A. Gray et al. [HPQCD and UKQCD Collaborations], Phys. Rev. D 72, 094507 (2005); T. Burch and C. Ehmann, Nucl. Phys. A 797, 33(2007); T.-W. Chiu et al. [TWQCD Collaboration], Phys. Lett. B 651, 171 (2007).

[6] F. Domingo, U. Ellwanger, M.A. Sanchis-Lozano, Phys. Rev. Lett. 103, 111802 (2009); A.A. Penin, Nucl. Phys. Proc. Suppl. 205, 256 (2010).

[7] K. Nakamura et al. (Particle Data Group), Journal of Physics G 37, 075021 (2010).

[8] S. Godfrey and J.L. Rosner, Phys. Rev. D 66, 014012 (2002); J.L. Rosner et al. (CLEO Collaboration), Phys. Rev. Lett. 95, 102003 (2005).

[9] M. Ablikim et al. (BES Collaboration), Phys. Rev. Lett. 104, 132002 (2010).

[10] J.L. Rosner et al. (CLEO Collaboration), Phys. Rev. Lett. 95, 102003 (2005); S. Dobbs et al. (CLEO Collaboration), Phys. Rev. Lett. 101, 182003 (2008).

[11] G.S. Adams et al. (CLEO Collaboration), Phys. Rev. D 80, 051106 (2009).

[12] B. Aubert et al. (BaBar Collaboration), Nucl. Instrum. Meth. A 479, 1 (2002).

[13] A. Abashian et al. (Belle Collaboration), Nucl. Instrum. Methods Phys. Res., Sect. A 479, 117 (2002).

[14] J.P. Lees et al. (BaBar Collaboration), arXiv:1102.4564, submitted to Phys. Rev. D(R) (2011).

[15] I. Adachi et al. (Belle Collaboration), arXiv:1103.3419v1.

[16] M. Artuso et al. (CLEO Collaboration), Phys. Rev. Lett. 94, 032001 (2005).

[17] S. Recksiegel and Y. Sumino, Phys. Lett. B 578, 369 (2004); B.A. Kniehl et al., Phys. Rev. Lett. 92, 242001 (2004); ibid. 104, 199901(E) (2010).

[18] A. Gray et al. (HPQCD and UKQCD Collaborations), Phys. Rev. D 72, 094507 (2005); T. Burch et al. (Fermilab and MILC Collaborations), Phys. Rev. D 81, 034508 (2010); S. Meinel, Phys. Rev. D 82, 114502 (2010).

[19] M.B. Voloshin, Sov. J. Nucl. Phys. 43, 1011 (1986).

[20] S. Godfrey, J. Phys. Conf. Ser. 9, 123 (2005).

[21] Y.P. Kuang and T.M. Yan, Phys. Rev. D 24, 2874 (1981); Y.P. Kuang, S.F. Tuan, and T.M. Yan, Phys. Rev. D 37, 1210 (1988); Y.P. Kuang and T.M. Yan, Phys. Rev. D 41, 155 (1990); S.F. Tuan, Mod. Phys. Lett. A 7, 3527 (1992).

[22] F. Butler et al. (CLEO Collaboration), Phys. Rev. D 49, 40 (1994).

[23] K.-F. Chen et al. (Belle Collaboration), Phys. Rev. Lett. 100, 112001 (2008).

[24] W.-S. Hou, Phys. Rev. D 74, 017504 (2006).

[25] R.E. Mitchell, arXiv:1102.3424v1. 